УДК 519.673

СFD-МОДЕЛИРОВАНИЕ СТАТИЧЕСКОГО СМЕСИТЕЛЯ ДЛЯ ОБЕССОЛИВАНИЯ НЕФТИ

\title{
CFD-MODELING OF A STATIC MIXER FOR OIL DESALTING
}

\author{
Р.Ф. Ахметов, А.Х. Мухаметьянова, Г.М. Сидоров, \\ Б.А. Яхин, А.Р. Набиева, Р.Ю. Кондратьев
}

Уфимский государственный нефтяной технический университет, г. Уфа, Российская Федерация

\section{Rustam F. Akhmetov, Albina H. Mukhametyanova, Georgij M. Sidorov, Bulat A. Yakhin, Albina R. Nabieva, Roman Yu. Kondratyev \\ Ufa State Petroleum Technological University, Ufa, Russian Federation e-mail: albina-m1996@mail.ru}

Аннотация. Одним из способов увеличения глубины обессоливания нефти является применение статических смесителей. Работа смесителей заключается в интенсивном перемешивании нефти и воды. Статические смесители широко применяются в процессах подготовки нефти за счет простоты конструкции и низкой стоимости.

Процесс численного анализа гидродинамики используется в статических смесителях для расчета скоростей потока, перепада давления и подбора оптимальной конструкции винтового закручивающего устройства.

Статья посвящена исследованию гидродинамики статического смесителя для обессоливания нефти в рабочих условиях, представлены результаты разработки модели для исследования гидродинамики работы статического смесителя с закручивающим устройством с применением CFD (Computational Fluid Dynamics). Приведены данные краткого обзора 
использования CFD-метода для расчета кинетической энергии турбулизации и перепада давления для статических смесителей, результаты исследования гидродинамики статического смесителя в рабочих условиях, моделирования процесса смешивания нефти и воды. Задача исследования заключается в изучении перепада давления и генерации турбулентной энергии в смесителях с различной геометрией закручивающего устройства. Моделирование проводилось с изменением геометрических размеров прорезей, а именно с изменением соотношения сторон прямоугольных прорезей закручивающего устройства нефти в статическом смесителе в 2-5 раз. В результате CFD-моделирования изучены изменения показателей турбулизации потока и перепад давления на смесителе в зависимости от соотношения сторон прямоугольных прорезей в закручивающемся устройстве.

В результате анализа экспериментальных данных показано, что с увеличением соотношения сторон прямоугольных прорезей закручивающего устройства нефти с 2 до 5 кинетическая энергия турбулизации увеличивается на 57,5 \%.

Использование CFD-моделирования позволяет моделировать процессы с высокой точностью, значительно сокращает время и материальные ресурсы на разработку высокоэффективных статических смесителей для обессоливания нефти.

Abstract. One of the ways to increase the depth of oil desalination is the use of static mixers. The work of the mixers consists in the intensive mixing of oil and water. Static mixers are widely used in oil treatment processes due to the simplicity of design and low cost.

The process of numerical analysis of hydrodynamics is used in static mixers to calculate flow rates, differential pressure and the selection of the optimal design of a screw twisting device.

The work is devoted to the study of the hydrodynamics of a static mixer for desalting oil under operation, the results of the development of a model for 
studying the hydrodynamics of a static mixer with a swirling device using CFD (Computational Fluid Dynamics) are presented. The data of a brief review of the use of the CFD method for calculating the kinetic energy of turbulization and pressure drop for static mixers, the results of a study of the hydrodynamics of a static mixer under operating conditions, and modeling the process of mixing oil and water are presented. The objective is to study the pressure drop and the generation of turbulent energy in mixers with different geometry of the swirling device. The simulation was carried out with a change in the geometric dimensions of the slots, namely, with a change in the aspect ratio of the rectangular slots of the oil swirling device in a static mixer by $2-5$ times. As a result of CFD modeling, the changes in flow turbulence and the pressure drop on the mixer depending on the aspect ratio of the rectangular slots in the swirling device were studied.

Ключевые слова: CFD; гидродинамика; моделирование; обессоливание; статический смеситель; твердотельная модель; закручивающее устройство; турбулизация; перепад

Key words: CFD; hydrodynamics; modeling; desalination; static mixer; solid state model; spinning device; turbulence; delta

На сегодняшний день актуальной проблемой является уменьшение содержания минеральных солей при подготовке нефти. Хлористые соли, в особенности хлориды кальция и магния, гидролизуются с образованием соляной кислоты, под действием которой происходит коррозия оборудования, в результате на внутренней поверхности трубопроводов, теплообменников в змеевиках нагревательных печей образуются отложения солей. Отложения в трубопроводах уменьшают их проходное сечение, что приводит к снижению производительности. Одним из способов увеличения глубины обессоливания нефти является применение статических смесителей [1-3]. Работа статических смесителей заключается в интенсивном перемешивании нефти и воды. Эти смесители широко 
применяются в процессах подготовки нефти за счет простоты конструкции и низкой стоимости.

За последние 20 лет с развитием вычислительной гидродинамики (Computational Fluid Dynamic) и увеличением вычислительных мощностей компьютеров стало возможным численно анализировать гидродинамику в статических смесителях для перемешивания различных компонентов. Так, в работе П. Пианко-Опрух, 3. Джаворски [4] представлен обзор применения средств вычислительной гидродинамики для расчета скоростей потока и перепада давления для статических смесителей SMX. B работе Ф. Ферон, Н. Саузе [5] проведено сравнение по перепаду давления в турбулентном режиме течения для трех статических смесителей фирмы SMX. М. Шабани, М. Ализадех, А. Мазахери [6, 7] разработали модель на основе вычислительной гидродинамики для смесителя-отстойника, согласно которой предложена оптимальная геометрия данного смесителя с более высокой эффективностью. 3. Джаворски, Х. Муразиевич [8] с помощью CFD-метода исследовали смешивание нефти и воды в смесителе Kenics. И. Гаспар, П. Текич [9] по результатам CFD-метода оптимизировали геометрию статического смесителя. Г. Чен, 3. Луи [10] продолжали изучение смесителей фирмы Kenics с использованием метода вычислительной гидродинамики о влиянии соотношения сторон и угла закрутки на перепад давления в смесителях. М. Стек, П. Синовиек $[11,12]$ разработали численную модель для статического смесителя фирмы Koflo для прогнозирования перепада давления при турбулентном течении.

В paботах [13-15] показано, что с помощью CFD-метода можно получить подробную информацию об эффективности перемешивания нефти с пресной водой и разработать конструкцию высокоэффективного статического смесителя для процесса обессоливания нефти и усовершенствовать газодинамический метод разделения углеводородных газов за счет подбора оптимальной конструкции винтового закручивающего устройства [16]. Опытно-промышленными испытаниями 
струйного гидравлического смесителя, разработанного на основе данных моделирования, подтверждена высокая эффективность в промысловых условиях и достоверность CFD-метода.

Большое количество работ посвящено применению вычислительной гидродинамики в статических смесителях с точки зрения интенсификации процесса, основываясь на характеристиках смешивания и массопереноса [17-21].

Данная статья посвящена исследованию гидродинамики статического смесителя для обессоливания нефти в рабочих условиях. Моделирование проводилось с изменением геометрических размеров тангенсальных прорезей, а именно с изменением соотношения сторон $\mathrm{a} / b$ прорези закручивающего устройства нефти в статическом смесителе в 2-5 раз.

\section{Объекты и методы исследования}

Для моделирования работы статического смесителя была построена твердотельная модель. Созданная в CAD-системе модель была импортирована в модуль ANSYS Design Modeler, где было произведено извлечение расчетного домена. Для создания расчетной сетки использовался модуль ANSYS Meshing. Для моделирования гидродинамики использовался инструмент ANSYS CFX.

Трехмерная твердотельная модель статического смесителя с закручивающим устройством представлена на рисунке 1.

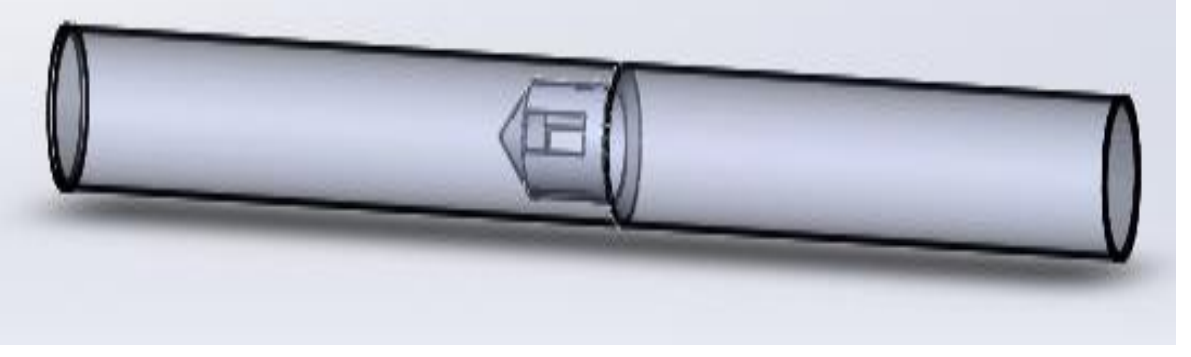

Рисунок 1. Твердотельная модель статического смесителя 
Твердотельная модель статического смесителя состоит из цилиндрического корпуса, внутри которого установлено закручивающее устройство. Закручивающее устройство состоит из конической заглушки, направленной острием навстречу потока, цилиндрического корпуса с прямоугольными прорезами и конического перехода.

Твердотельная модель закручивающего устройства с прорезями представлена на рисунке 2.

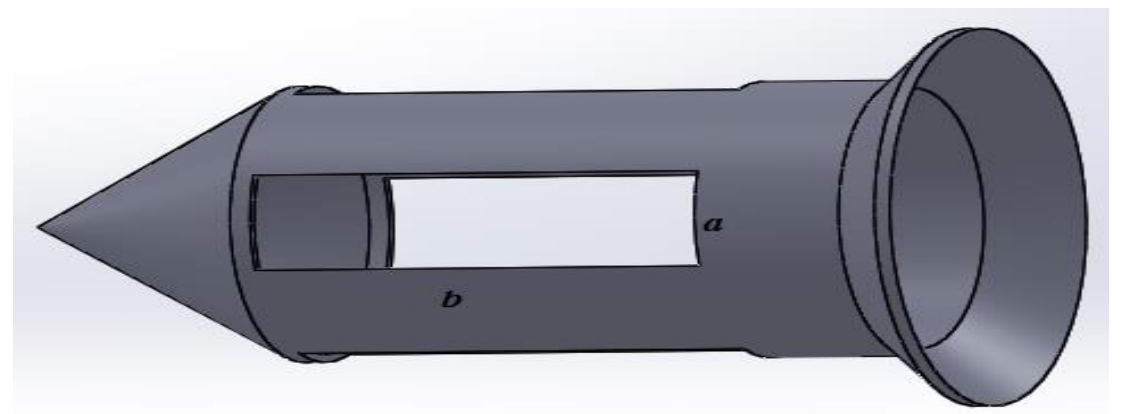

Рисунок 2. Твердотельная модель закручивающего устройства

В гидродинамических расчетах была использована полуэмпирическая $k-\varepsilon$ модель турбулентности, где $k$ учитывает кинетическую энергию турбулентности и $\varepsilon-$ скорость диссипации турбулентности. Согласно работам [22-24], использование турбулентных моделей приводит к более точным результатам, чем модель ламинарного потока для исследования сложных процессов взаимодействия нефти и воды в статических смесителях.

Количество элементов конечно-элементной сетки составило 626878. Расчетная сетка сгенерирована в модуле ANSYS Meshing.

\section{Результаты и их обсуждение}

Одним из способов уменьшения содержания хлористых солей при подготовке нефти является интенсивная промывка нефти пресной водой. Высокая степень диспергирования воды в нефти достигается за счет турбулизации потока. 
Моделирование процесса турбулизации проводилось при скорости потока жидкости, поступающей в смеситель, равной 2,5м/с.

В результате CFD-моделирования анализировали показатели турбулизации потока и перепада давления в смесителе в зависимости от соотношения сторон $a / b$ в закручивающемся устройстве.

Результаты симуляции работы статического смесителя приведены на рисунке 3.

Из анализа рисунка 3 видно, что при соотношении сторон прорези $a / b=5$ распределение кинетической энергии турбулизации потока жидкости в статическом смесителе с закручивающим устройством увеличивается.

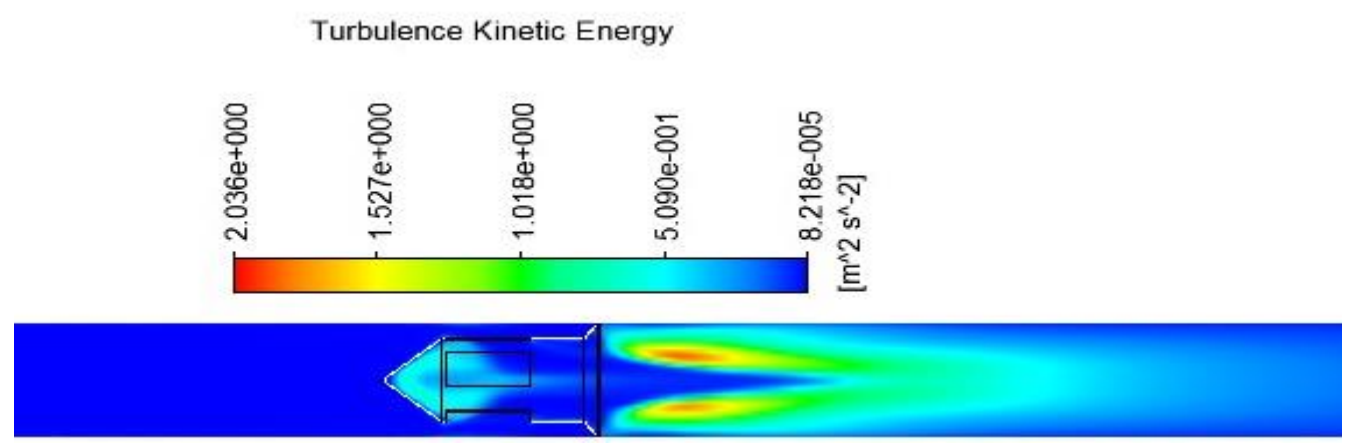

при соотношении $a / b=2$
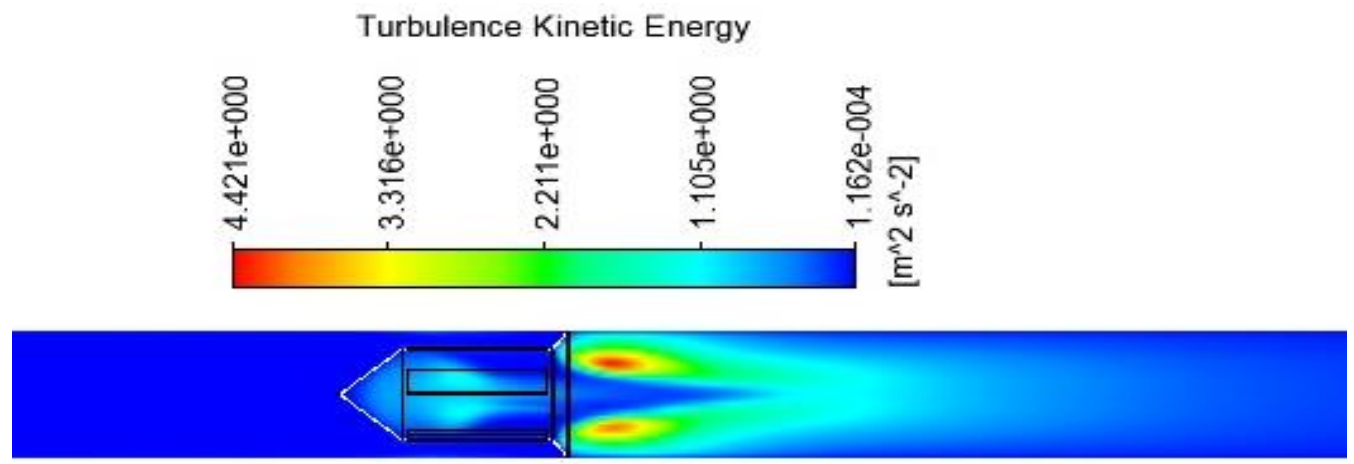

при соотношении $a / b=5$

Рисунок 3. Распределение кинетической энергии турбулизации потока жидкости в статическом смесителе с закручивающим устройством 
На рисунке 4 приведено сравнение скорости потока при разных соотношениях сторон $a$ и $b$ в закручивающем устройстве.
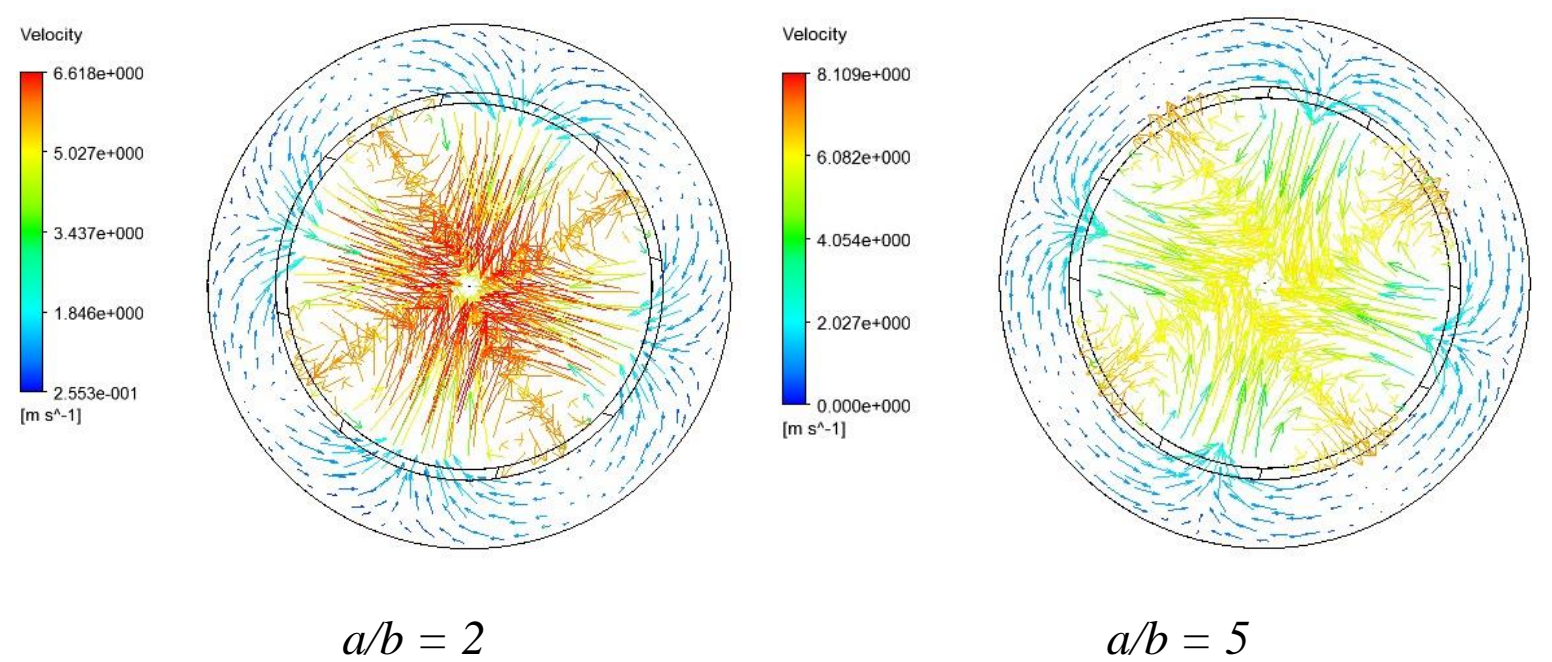

Рисунок 4. Распределение скорости потока в закручивающем устройстве при разных соотношениях $a / b$

При соотношении $a / b=5$ распределение скорости потока в закручивающем устройстве более равномерно по всему сечению смесителя (рисунок 4), что положительно влияет на интенсивность перемешивания нефти и пресной воды.

Перепад давления в смесителе возникает при прохождении жидкости через прорези закручивающего устройства. Величина перепада давления зависит от размера прорези и соотношения сторон. Увеличение перепада давления приводит к повышению турбулизации потока и интенсивности перемешивания нефти с пресной водой при обессоливании.

График изменения перепада давления в закручивающем устройстве приведен на рисунке 5. 


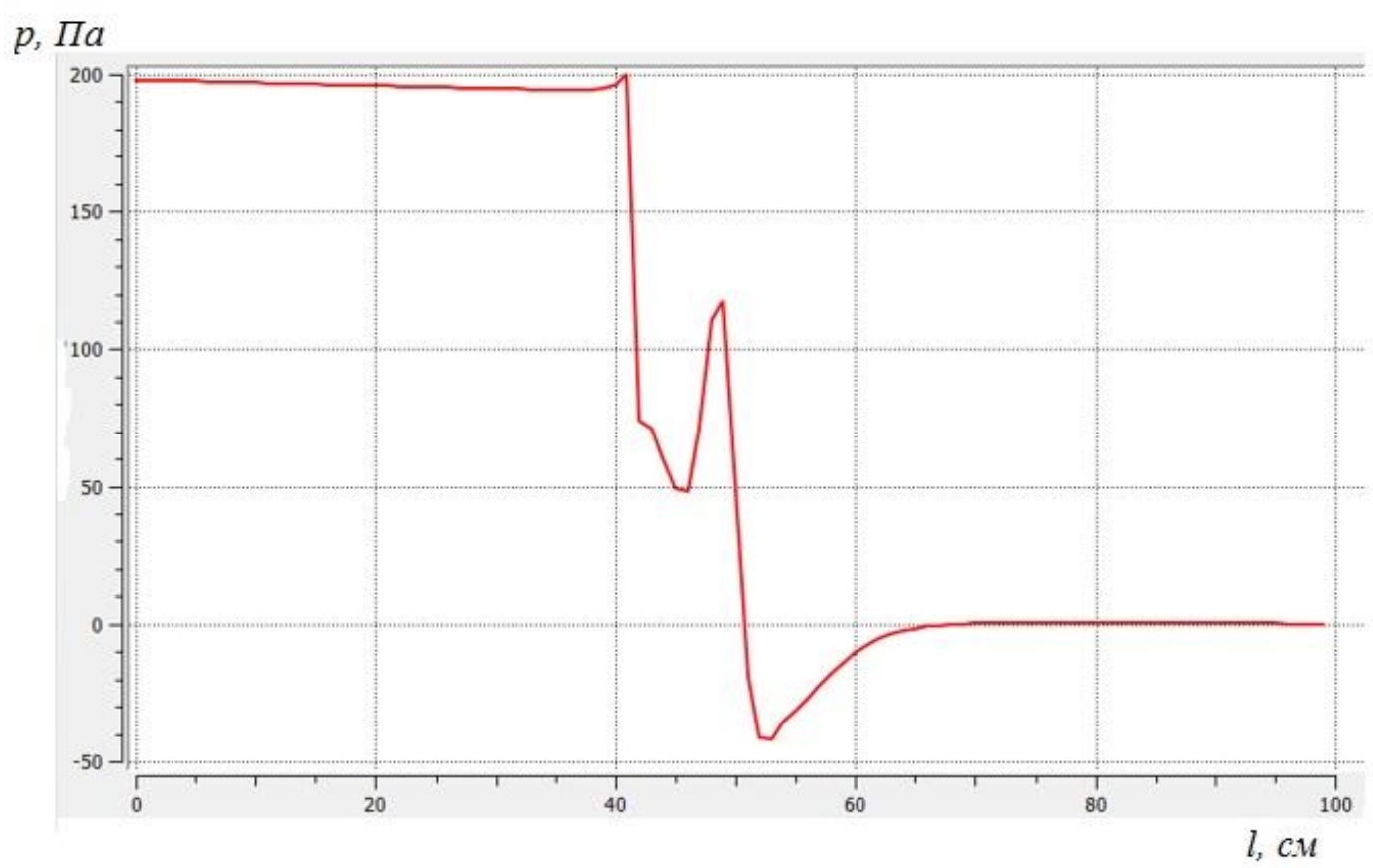

Рисунок 5. График изменения перепада давления в закручивающем устройстве нефти

Из анализа рисунка 5 видно, что наибольшее давление перед вводом жидкости в закручивающее устройство. При вводе жидкости в кольцевое сечение между корпусом смесителя и закручивающим устройством скорость потока увеличивается из-за уменьшения площади сечения, следовательно, снижается давление. Скорость потока также увеличивается, когда жидкость проходит через прорези закручивающего устройства, и, как следствие, снижается давление. При прохождении жидкости внутри закручивающего устройства и на выходе в корпус смесителя скорость потока снижается, следовательно, увеличивается перепад давления.

На рисунке 6 представлены экспериментальные данные (перепад давления кинетическая и энергия турбулизации) в зависимости от соотношения сторон $a / b$ в закручивающем устройстве. 


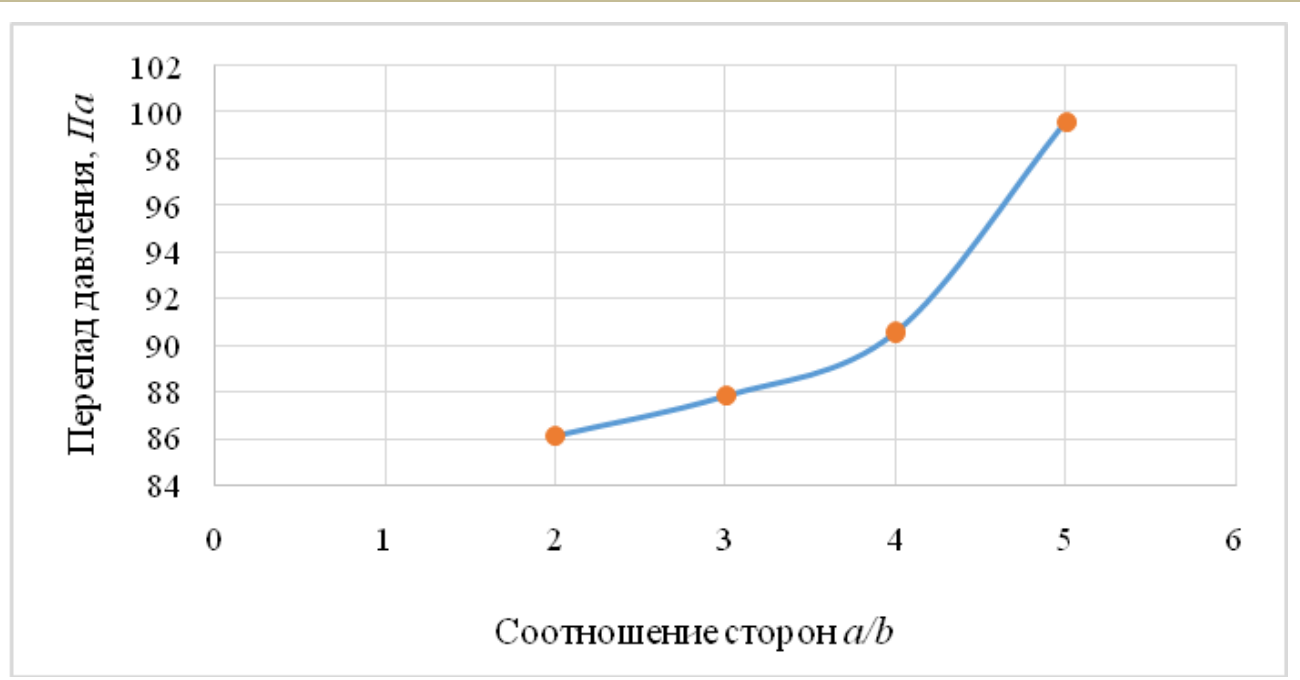

a)

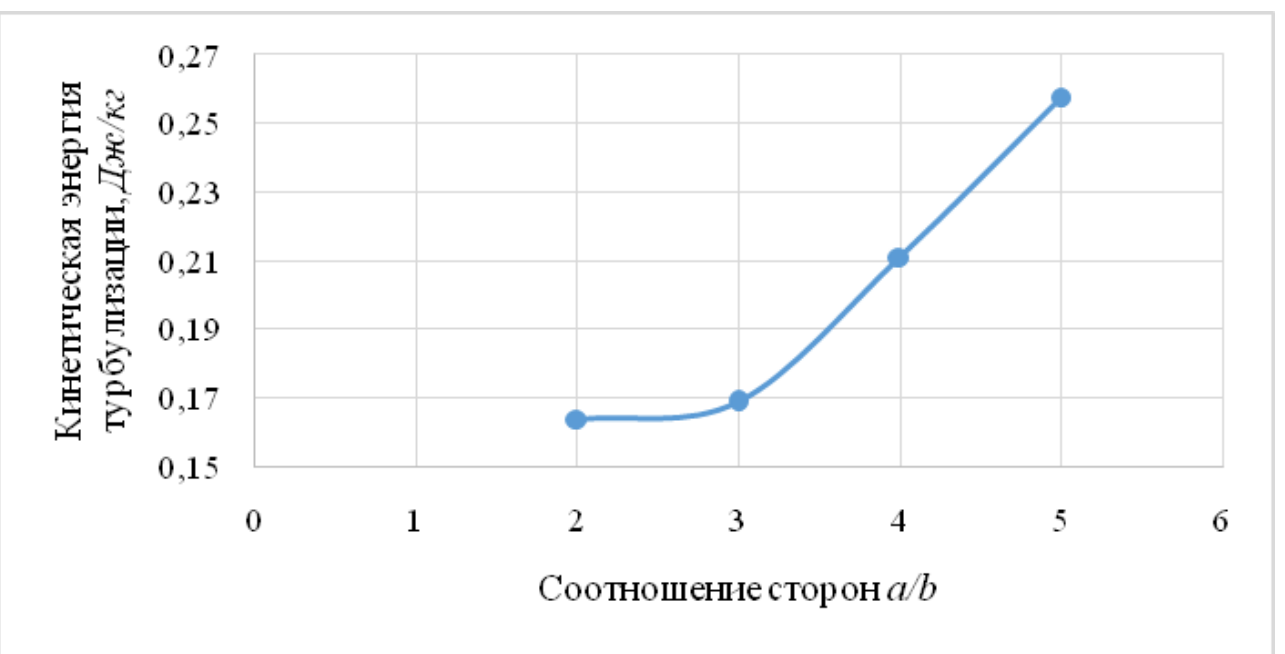

б)

Рисунок 6. Результаты CFD-моделирования:

график зависимости перепада давления на устройстве от соотношения сторон $a / b(a)$ и график зависимости кинетической энергии турбулизации от соотношения сторон $a / b$ (б)

Для обеспечения высокой степени диспергирования воды в нефти и повышения эффективности обессоливания необходимо обеспечить интенсивную турбулизацию потока. Анализ экспериментальных данных, приведенных на рисунке 6, показывает, что с увеличением соотношения сторон $a / b$ прорези закручивающего устройства нефти с 2 до 5 кинетическая энергия турбулизации увеличивается с 0,1636 до 
0,2577 Дж/кг (на 57,5 \%). Это оказывает положительное влияние на процесс обессоливания нефти, но при этом повышается и перепад давления в закручивающем устройстве с 86,12 до 99,57 Па (на 15,6 \%).

\section{Вывод}

Использование современных методов вычислительной гидродинамики и проведение сравнительного анализа влияния соотношения сторон прямоугольных прорезей закручивающего устройства в статическом смесителе на турбулизацию потока позволяет моделировать процессы смешения нефти и воды с высокой точностью, что значительно сокращает время и материальные ресурсы на разработку высокоэффективных статических смесителей с закручивающим устройством для обессоливания нефти.

При конструировании статического смесителя соотношение размеров прямоугольных прорезей закручивающего устройства необходимо выбирать между показателями наибольшей кинетической энергии турбулизации и допустимым перепадом давления в технологической цепочке с учетом конкретных условий работы установки подготовки нефти.

\section{Список используемых источников}

1. Жолобова Г.Н., Хисаева Е.М., Сулейманов А.А., Галиакбаров В.Ф. Совершенствование процессов подготовки нефти // Электронный научный журнал «Нефтегазовое дело». 2010. № 1. C. 76-83. URL: http://ogbus.ru/files/ogbus/authors/Zholobova/Zholobova_1.pdf (дата обращения: 15.11.2019).

2. Хуторянский Ф.М. Современное состояние и варианты совершенствования установок подготовки нефти. Основные направления перспективных научно-исследовательских работ в области глубокого обессоливания нефти // Технологии нефти и газа. 2010. № 6. С. 33-39. 
3. Кондрашева Н.К., Дубовиков О.А., Иванов И.И., Зырянова О.В. К вопросу о предварительной подготовке нефти к первичной переработке // Записки Горного института. 2014. Т. 201. С. 21-29.

4. Pianko-Opruch P., Jaworski Z. CFD Modeling of Liquid-Liquid Flow in a SMX Static Mixer // Polish Journal of Chemical Technology. 2009. Vol. 3. P. 41-49. DOI: 10.2478/v10026-009-0034-x.

5. Theron F., Le Sauze N. Comparison between Three Static Mixers for Emulsification in Turbulent Flow // International Journal of Multiphase Flow. 2011. Vol. 5. P. 488-500. DOI: 10.1016/J.IJMULTIPHASEFLOW.2011.01.004.

6. Shabani M., Alizadeh M., Mazahery A. Fluid Flow Characterization of Liquid-Liquid Mixing in Mixer-Settler // Engineering with Computers. 2011. Vol. 4. P. 373-379. DOI: 10.1007/s00366-011-0209-y.

7. Shabani M., Mazahery A. Evaluation of the Effect of Mixer Settler Baffles on Liquid-Liquid Extraction via CFD Simulation // UPB Scientific Bulletin Series D. 2011. Vol. 73. Issue 4. P. 55-63. URL: https://www.scientificbulletin.upb.ro/rev_docs_arhiva/full95010.pdf (дата обращения: 15.11.2019).

8. Jaworski Z., Murasiewicz H. Numerical and Experimental Studies of Liquid-Liquid Mixing in a Kenics Static Mixer // Proceedings the 14th European Conference on Mixing. Warsaw, Poland. 2012. Vol. 14. P. 181-186. URL: http://mixing14.eu/p/mixing14eu_28.pdf (дата обращения: 17.11.2019).

9. Gaspar I., Tekic P., Koris A., Krisztina A., Popovic S., Vatai G. CFD and Laboratory Analysis of Axial Cross-Flow Velocity in Porous Tube Packed with Differently Structured Static Turbulence Promoters // Chemical Industry. 2015. Vol. 69. P. 713-718. DOI: 10.2298/HEMIND140312001G.

10. Chen G., Liu Z. Numerical Research of Pressure Drop in Kenics Static Mixer // Advanced Materials Research. 2013. P. 547-551. DOI: 10.4028/www.scientific.net/AMR.694-697.547. 
11. Stec M., Synowiec P. Numerical Method Effect on Pressure Drop Estimation in the Koflo Static Mixer // Inzynieria I Aparatura Chemiczna. 2015. Vol. 2. P. 48-50.

12. Stec M., Synowiec P. Analysis of the Pressure Drop Calculation Method Impact on the Accuracy of the Experimental Results in the Koflo Static Mixer // Inzynieria I Aparatura Chemiczna. 2015. Vol. 4. P. 201-203.

13. Сидоров Г.М., Яхин Б.А., Ахметов Р.Ф. Моделирование работы статического смесителя (нефть-вода) для обессоливания нефти и опытнопромышленное испытание // Успехи современного естествознания. 2017. № 2. C. 152-156.

14. Кашапова Л.А., Марушкин А.Б., Сидоров Г.М., Яхин Б.А. Опыт использования струйных гидравлических смесителей при подготовке нефти на промыслах Татарстана // Мир нефтепродуктов. Вестник нефтяных компаний. 2017. № 11. С. 37-39.

15. Пат. 180014 РФ, МПК В 01 F 5/06. Струйный смеситель / Г.М. Сидоров, Р.Н. Бахтизин, Б.А. Яхин, Р.3. Нургалиев. 2018106628, Заявлено 21.02.2018; Опубл. 03.05.2018. Бюл. 16.

16. Ахметов Р.Ф., Сидоров Г.М., Рахимов М.Н., Беркань В.О. Совершенствование конструкции винтового закручивающего устройства методом CFD-анализа // Фундаментальные исследования. 2015. № 11-4. C. 647-653.

17. Yang A., Hsieh Y., Kuo L., Tseng L., Liao S. A Novel Vortex Mixer Actuated One-Shot Electricity-Free Pumps // Chemical Engineering Journal. 2013. Vol. 228. P. 882-888. DOI: 10.1016/j.cej.2013.05.053.

18. Abdolkarimi V., Ganji H. CFD Modeling of Two Immiscible Fluids Mixing in a Commercial Scale Static Mixer // Brazilian Journal of Chemical Engineering. 2014. Vol. 4. P. 949-957. DOI: 10.1590/0104$6632.20140314 \mathrm{~s} 00002857$. 
19. Konopacki M., Kordas M., Fijalkowski K., Rakoczy R. Computational Fluid Dynamics and Experimental Studies of a New Mixing Element in a Static Mixer as a Heat Exchanger // Chemical and Process Engineering. 2015. Vol. 6. P. 59-72. DOI: 10.1515/cpe-2015-0005.

20. Chanem A., Lemenand T., Valle D.D., Peerhossaini H. Static Mixers: Mechanisms, Applications and Characterization Methods - A Review // Chemical Engineering Research and Design. 2014. Vol. 92. P. 205-228. DOI: 10.1016/j.cherd.2013.07.013.

21. Vasilev M.P., Abiev R.Sh. Turbulent Droplets in a Flow Type Apparatuses - New Type Static Disperser // Chemical and Process Engineering. 2018. Vol. 349. P. 646-661.

22. Irfan M., Kailash B., Subramanya G. CFD Analysis of a Single Phase Mixing of Fluids without the Aid of Stirrers // International Research Journal of Engineering and Technology. 2015. Vol. 2. Issue 9. P. 134-137.

23. Abdulmumuni A., Marhaendrajana T., Bindar Y. Application of CFD for Numerical Analysis of Liquid-Liquid Mixing in T-Shape Mixer Using Ansys Fluent // Preprints. 27.07.2018. URL: https://www.preprints.org/manuscript/ 201807.0548/v1 (дата обращения: 19.11.2019). DOI: 10.20944/preprints201807.0548.v1.

24. Murasiewicz H., Zakrzewska B. Large Eddy Simulation of Turbulent Flow and Heat Transfer in a Kenics Static Mixer // Chemical and Process Engineering. 2019. Vol. 40. P. 87-99. DOI: 10.24425/cpe.2019.126103.

\section{References}

1. Zholobova G.N., Khisaeva E.M., Suleimanov A.A., Galiakbarov V.F. Sovershenstvovanie protsessov podgotovki nefti [Improving Oil Preparation Processes]. Elektronnyi nauchnyi zhurnal «Neftegazovoe delo»-Electronic Scientific Journal «Oil and Gas Business», 2010, No. 1, pp. 76-83. URL: http://ogbus.ru/files/ogbus/authors/Zholobova/Zholobova_1.pdf (accessed 15.11.2019). [in Russian]. 
2. Khutoryanskii F.M. Sovremennoe sostoyanie i varianty sovershenstvovaniya ustanovok podgotovki nefti. Osnovnye napravleniya perspektivnykh nauchno-issledovatel'skikh rabot $\mathrm{V}$ oblasti glubokogo obessolivaniya nefti [The Current State and Ways of Retrofitting of Crude Treatment Plants. The Main Tendencies of Advanced Research Work in Deep Crude Desalting]. Tekhnologii nefti $i$ gaza - Science and Technology of Hydrocarbons, 2010, No. 6, pp. 33-39. [in Russian].

3. Kondrasheva N.K., Dubovikov O.A., Ivanov I.I., Zyryanova O.V. K voprosu o predvaritel'noi podgotovke nefti $\mathrm{k}$ pervichnoi pererabotke [On the Issue of Preliminary Preparation of Oil for Primary Processing]. Zapiski Gornogo instituta - Journal of Mining Institute, 2014, Vol. 201, pp. 21-29. [in Russian].

4. Pianko-Opruch P., Jaworski Z. CFD Modeling of Liquid-Liquid Flow in a SMX Static Mixer. Polish Journal of Chemical Technology, 2009, Vol. 3, pp. 41-49. DOI: 10.2478/v10026-009-0034-х.

5. Theron F., Le Sauze N. Comparison between Three Static Mixers for Emulsification in Turbulent Flow. International Journal of Multiphase Flow, 2011, Vol. 5, pp. 488-500. DOI: 10.1016/ J.IJMULTIPHASEFLOW.2011.01.004.

6. Shabani M., Alizadeh M., Mazahery A. Fluid Flow Characterization of Liquid-Liquid Mixing in Mixer-Settler. Engineering with Computers, 2011, Vol. 4, pp. 373-379. DOI: 10.1007/s00366-011-0209-y.

7. Shabani M., Mazahery A. Evaluation of the Effect of Mixer Settler Baffles on Liquid-Liquid Extraction via CFD Simulation. UPB Scientific Bulletin Series D, 2011, Vol. 73, Issue 4, pp. 55-63. URL: https://www.scientificbulletin.upb.ro/rev_docs_arhiva/full95010.pdf (accessed 15.11.2019). 
8. Jaworski Z., Murasiewicz H. Numerical and Experimental Studies of Liquid-Liquid Mixing in a Kenics Static Mixer. Proceedings the 14th European Conference on Mixing. Warszawa, Poland, 2012, Vol. 14, pp. 181-186. URL: http://mixing14.eu/p/mixing14eu_28.pdf (accessed 17.11.2019).

9. Gaspar I., Tekic P., Koris A., Krisztina A., Popovic S., Vatai G. CFD and Laboratory Analysis of Axial Cross-Flow Velocity in Porous Tube Packed with Differently Structured Static Turbulence Promoters. Chemical Industry, 2015, Vol. 69, pp. 713-718. DOI: 10.2298/HEMIND140312001G.

10. Chen G., Liu Z. Numerical Research of Pressure Drop in Kenics Static Mixer. Advanced Materials Research, 2013, pp. 547-551. DOI: 10.4028/www.scientific.net/AMR.694-697.547.

11. Stec M., Synowiec P. Numerical Method Effect on Pressure Drop Estimation in the Koflo Static Mixer. Inzynieria I Aparatura Chemiczna, 2015, Vol. 2, pp. 48-50.

12. Stec M., Synowiec P. Analysis of the Pressure Drop Calculation Method Impact on the Accuracy of the Experimental Results in the Koflo Static Mixer. Inzynieria I Aparatura Chemiczna, 2015, Vol. 4, pp. 201-203.

13. Sidorov G.M., Yakhin B.A., Akhmetov R.F. Modelirovanie raboty staticheskogo smesitelya (neft'-voda) dlya obessolivaniya nefti i opytnopromyshlennoe ispytanie [Modeling Process of Static Mixers (Oil-Water) for Desalting Crude Oil and Pilot-Industrial Testing]. Uspekhi sovremennogo estestvoznaniya - Advances in Current Natural Sciences, 2017, No. 2, pp. 152156. [in Russian].

14. Kashapova L.A., Marushkin A.B., Sidorov G.M., Yakhin B.A. Opyt ispol'zovaniya struinykh gidravlicheskikh smesitelei pri podgotovke nefti na promyslakh Tatarstana [Experience of Using Jetting Hydraulic Mixer for Treatment Crude Oil in Tatarstan]. Mir nefteproduktov. Vestnik neftyanykh kompanii - World of Oil Products. The Oil Companies' Bulletin, 2017, No. 11, pp. 37-39. [in Russian]. 
15. Sidorov G.M., Bakhtizin R.N., Yakhin B.A., Nurgaliev R.Z. Struinyi smesitel' [Jet Mixer]. Patent RF, No. 180014, 2018. [in Russian].

16. Akhmetov R.F., Sidorov G.M., Rakhimov M.N., Berkan' V.O. Sovershenstvovanie konstruktsii vintovogo zakruchivayushchego ustroistva metodom CFD-analiza [Improving the Design of Helical Vortex Generator with CFD-Analysis]. Fundamental'nye issledovaniya - Fundamental Research, 2015, No. 11-4, pp. 647-653. [in Russian].

17. Yang A., Hsieh Y., Kuo L., Tseng L., Liao S. A Novel Vortex Mixer Actuated One-Shot Electricity-Free Pumps. Chemical Engineering Journal, 2013, Vol. 228, pp. 882-888. DOI: 10.1016/j.cej.2013.05.053.

18. Abdolkarimi V., Ganji H. CFD Modeling of Two Immiscible Fluids Mixing in a Commercial Scale Static Mixer. Brazilian Journal of Chemical Engineering, 2014, Vol. 4, pp. 949-957. DOI: 10.1590/01046632.20140314s00002857.

19. Konopacki M., Kordas M., Fijalkowski K., Rakoczy R. Computational Fluid Dynamics and Experimental Studies of a New Mixing Element in a Static Mixer as a Heat Exchanger. Chemical and Process Engineering, 2015, Vol. 6, pp. 59-72. DOI: 10.1515/cpe-2015-0005.

20. Chanem A., Lemenand T., Valle D.D., Peerhossaini H. Static Mixers: Mechanisms, Applications and Characterization Methods - A Review. Chemical Engineering Research and Design, 2014, Vol. 92, pp. 205-228. DOI: 10.1016/j.cherd.2013.07.013.

21. Vasilev M.P., Abiev R.Sh. Turbulent Droplets in a Flow Type Apparatuses - New Type Static Disperser. Chemical and Process Engineering, 2018, Vol. 349, pp. 646-661.

22. Irfan M., Kailash B., Subramanya G. CFD Analysis of a Single Phase Mixing of Fluids without the Aid of Stirrers. International Research Journal of Engineering and Technology, 2015, Vol. 2, Issue 9, pp. 134-137. 
23. Abdulmumuni A., Marhaendrajana T., Bindar Y. Application of CFD for Numerical Analysis of Liquid-Liquid Mixing in T-Shape Mixer Using Ansys Fluent. Preprints. 27.07.2018. Available at: https://www.preprints.org/ manuscript/201807.0548/v1 (accessed 19.11.2019). DOI: 10.20944/preprints201807.0548.v1.

24. Murasiewicz H., Zakrzewska B. Large Eddy Simulation of Turbulent Flow and Heat Transfer in a Kenics Static Mixer. Chemical and Process Engineering, 2019, Vol. 40, pp. 87-99. DOI: 10.24425/cpe.2019.126103.

\section{Сведения об авторах}

\section{About the authors}

Ахметов Рустам Фаритович, канд. техн. наук, доцент кафедры «Технология нефти и газа», УГНТУ, г. Уфа, Российская Федерация

Rustam F. Akhmetov, Candidate of Engineering Sciences, Assistant Professor of Oil and Gas Processing Department, USPTU, Ufa, Russian Federation

e-mail: ahmetov.rustam2011@gmail.com

Мухаметьянова Альбина Халитовна, магистрант кафедры «Технология нефти и газа», УГНТУ, г. Уфа, Российская Федерация

Albina H. Mukhametyanova, Undergraduate Student of Oil and Gas Processing Department, USPTU, Ufa, Russian Federation

e-mail: albina-m1996@mail.ru

Сидоров Георгий Маркелович, д-р техн. наук, профессор кафедры «Технология нефти и газа», УГНТУ, г. Уфа, Российская Федерация

Georgij M. Sidorov, Doctor of Engineering Sciences, Professor of Oil and Gas Processing Department, USPTU, Ufa, Russian Federation e-mail: kaskad@ufanet.ru 
Яхин Булат Ахметович, аспирант кафедры «Разработка и эксплуатация нефтяных и газонефтяных месторождений», УГНТУ, г. Уфа, Российская Федерация

Bulat A. Yakhin, Post-graduate Student of Oil and Gas \& Oil Field of Development and Operation Department, USPTU, Ufa, Russian Federation e-mail: bulattrading@yahoo.com

Набиева Альбина Римовна, канд. техн. наук, доцент кафедры «Технология нефти и газа», УГНТУ, г. Уфа, Российская Федерация

Albina R. Nabieva, Candidate of Engineering Sciences, Assistant Professor of Oil and Gas Processing Department, USPTU, Ufa, Russian Federation

e-mail: albena90@mail.ru

Кондратьев Роман Юрьевич, студент кафедры «Газохимия и моделирование химико-технологических процессов», УГНТУ, г. Уфа, Российская Федерация

Roman Yu. Kondratyev, Student of Gas Chemistry and Modeling of Chemical Engineering Processes Department, USPTU, Ufa, Russian Federation e-mail: rku-10@mail.ru 